PlastOx 2007 (2009) 221-236

(C) EDP Sciences, 2009

DOI: $10.1051 /$ ptox/2009016

\title{
Influence d'un état mécanique sur la réactivité de surface en milieux aqueux des métaux c.f.c.
}

\author{
X. Feaugas, J. Creus, R. Sabot, H. El Alami, D. Large, M. Sahal, \\ C. Savall et C. Huvier \\ Laboratoire d'Étude des Matériaux en Milieux Agressifs, EA3167, Université de La Rochelle, \\ Avenue Michel Crépeau, 17042 La Rochelle Cedex 01, France
}

\begin{abstract}
Résumé. Nous démontrons, à travers ce travail de synthèse, l'importance de l'état métallurgique et en particulier de l'état de déformation sur la réactivité de la surface d'un solide en regards d'un environnement donné. Cette préoccupation, rarement abordée, est cependant essentielle dans de nombreux domaines tels que la corrosion sous contrainte, la fragilisation par l'hydrogène, la protection d'un environnement, la durabilité des revêtements anti-corrosion, les biomatériaux,... L'importance d'une démarche expérimentale rigoureuse est soulignée, ainsi que la nécessité de prendre en compte les diverses expressions d'un champ mécanique dans une approche de son influence sur les processus d'adsorption et de transfert de charges.
\end{abstract}

\section{INTRODUCTION}

La corrosion sous contrainte (CSC) des métaux c.f.c. est un mode d'endommagement structural difficile à modéliser. En effet, il implique l'interaction entre des paramètres chimiques et électrochimiques, liés à la nature du métal et au milieu, des paramètres caractéristiques de la structure macroscopique et microscopique du métal, et des paramètres mécaniques liés aux contraintes résiduelles ou appliquées au métal. Cette dégradation fait intervenir deux types de réactions opérant conjointement : des réactions anodiques impliquant les phénomènes de dissolution-repassivation, et des réactions cathodiques impliquant les effets de l'hydrogène. Trois mécanismes principaux sont relatés dans la littérature: les interactions hydrogène-plasticité [1,2], la rupture du film passif [3-6] et le mécanisme de mobilité de surface $[7,8]$. Bien que l'effet de l'environnement sur le comportement mécanique soit bien documenté, l'influence de la déformation plastique sur les processus de dissolution et d'adsorption n'est pas clairement établie, peu de travaux traitant de cet effet [9-11]. Il est reconnu que la corrosion sous contrainte correspond à des effets de synergie entre les processus de corrosion et le champ mécanique en pointe de fissure. Par conséquent, il semble important d'identifier l'influence du comportement élastoplastique des métaux sur les cinétiques électrochimiques liées aux processus de corrosion (adsorption, dissolution, passivation). Dans le cas particulier des processus de dissolution, quelques études de traction in-situ en milieux acides ont montré une augmentation de la densité de courant puis une diminution de celle-ci lorsque la déformation plastique augmente $[9,11-15]$. Ce résultat important peut être interprété de multiples façons : une augmentation de l'activité de la dissolution sur les marches associées à l'émergence des lignes de glissement, une augmentation de la rugosité, une réduction des distances de diffusion en surface, une modification de l'entropie du système associée à la présence de dislocations et d'un état de contrainte,... [11, 12]. Les tentatives d'interprétation des résultats in-situ ne distinguent que rarement les effets de la contrainte de ceux des défauts développés par la plasticité. Enfin, l'émergence des bandes de glissement conduit à une modification de la rugosité et à la rupture du film passif au cours de la sollicitation. Il est donc impossible de tirer des conclusions de tels résultats sans considérer une décomposition des effets associés à la déformation plastique en termes de contrainte, de dislocations, du champ de contrainte et de déformation des dislocations, d'émergence des dislocations, de modification de la rugosité et de stabilité du film passif lors de la formation de bandes de 
(a)

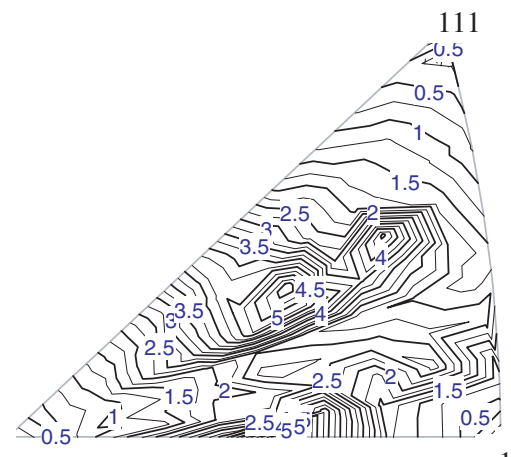

(b)

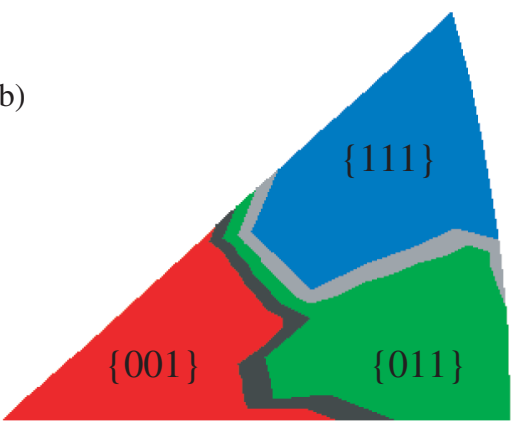

101

Figure 1. Densité (nombre de marches en $\mathrm{nm}^{-1}$ ) (a) et nature des terrasses (b) en fonction de l'orientation de la normale à la surface définie dans une figure de pôles inverses (BASALC program system Copyright K. Hermann 1991).

déformation. Dans le présent écrit, nous nous attacherons à faire la synthèse de récents travaux conduits dans cet objectif sur des métaux c.f.c. (essentiellement le nickel) [16-25]. Ainsi différentes méthodes expérimentales $e x$-situ et in-situ ont été développées dans le but d'effectuer des mesures précises de densité de courant et de déformation sur des états de surface reproductibles. En parallèle, une étude la plus exhaustive possible des différentes sources d'hétérogénéité de surface associées à la plasticité a été conduite en microscopie électronique en transmission (MET) et en microscopie à force atomique (AFM). La corrélation entre les défauts structuraux et la réactivité de surface a permis de clarifier un certain nombre d'effets de la contrainte et de la déformation plastique sur les processus de corrosion que sont la dissolution et l'adsorption de différentes espèces.

\section{QUELQUES REMARQUES SUR LA NATURE DE LA SURFACE REACTIVE}

L'organisation des atomes qui conduit à définir une structure cristallographique propre à un métal donné résulte d'un principe de minimisation de son énergie libre. Dans le même esprit, afin de minimiser l'énergie de surface, un métal va généralement s'organiser en surface sous la forme de terrasses constituées de faces de plus faible énergie (111), (110), (100) (fig. 1) [26]. Ainsi, il existe pour une orientation de la surface donnée, une multitude de marches mono-atomiques et de crans (fig. 2a) qui constituent des sites particuliers au sens où les atomes comme les électrons de valences associés à ceuxci sont plus faiblement liés au cristal que les sites propres aux terrasses. La densité de marches est fonction de la nature du plan de plus faible énergie constituant les terrasses et de l'orientation de la surface (fig. 1). Notons que la décomposition en terrasses, marches et coins n'a plus de sens lorsque les terrasses ont une largeur voisine de la distance inter-atomique (forte densité de marches, figure 1a). L'émergence d'une dislocation en surface, conduit à la formation d'une marche dont la hauteur est fonction du vecteur de burgers et de l'orientation de la ligne vis-à-vis de la normale à cette surface (fig. 2b). Ainsi la présence de dislocations au sein du cristal conduit en surface à une modification marquée de la densité de marches et de crans. L'ensemble des sites supplémentaires associés à la reconstruction de la surface et à la présence de dislocations favorise la réactivité de la surface, c'està-dire la potentialité qu'a celle-ci à se dissoudre (extraction d'atomes, d'électrons, ...) ou à adsorber certaines espèces en solution (hydrogène, hydroxyde,...). Dans les métaux c.f.c., les dislocations produites par une déformation plastique se distribuent rapidement de façon hétérogène (fig. 2c). Chaque taux de déformation plastique permet le développement d'une microstructure caractéristique d'un stade d'écrouissage en traction [27, 30, 31]. 

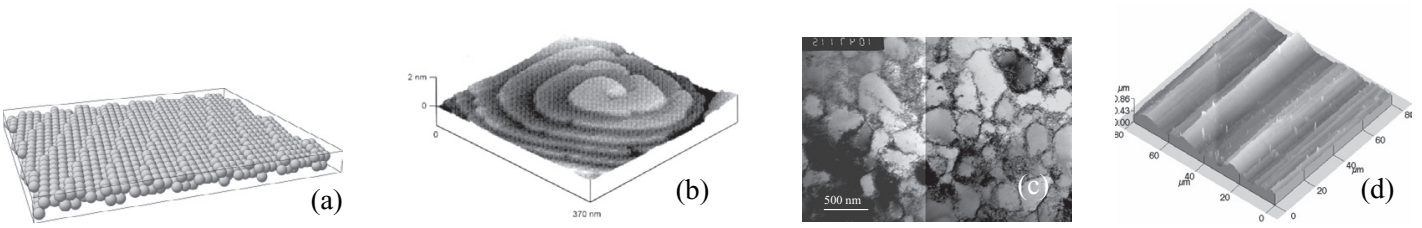

Figure 2. Hétérogénéités de surface à différentes échelles : marches et terrasses $\{111\}$ [25] (a), marches associées à l'émergence d'une dislocation hélicoïdale sur une surface \{111\} de l'argent [29] (b), cellules de dislocation dans le nickel [27] (c), émergence de bandes de déformation (stade II d'un monocristal de nickel orienté en glissement simple) [28] (d).

Le stade I est ainsi associé au glissement plan/simple qui développe une distribution de dislocations relativement homogène. Le glissement multiple et le glissement dévié apparaissent durant le stade II, pour des déformations plus importantes. Des structures hétérogènes se forment alors : enchevêtrements, murs et cellules de dislocations (fig. 2c). Cette répartition hétérogène des dislocations peut être décrite par un schéma composite caractérisé par une «phase dure»: zones de forte densité de dislocations $\left(\rho_{\mathrm{w}}\right)$ contenues dans les murs des cellules, et une «phase molle»: zones de faible densité de dislocations $\left(\rho_{\mathrm{c}}\right)$ contenues dans les zones inter-murs.

Le stade III, intervenant généralement au-delà de $10 \%$ de déformation plastique, est généralement attribué à la généralisation du glissement dévié. Ce dernier favorise les processus d'annihilation, induisant ainsi une diminution de la densité des dislocations dans la phase «dure ». L'hétérogénéité de déformation sera définie, par la suite, par une fraction de phase «dure» $f_{w}$. L'ense mble de ces caractéristiques sera utilisé pour établir une corrélation entre les défauts cristallographiques de surface, engendrés par les dislocations, et les processus électrochimiques associés à la corrosion. Notons que la densité élevée de dislocations, présente au sein des murs, est à l'origine de forte concentration de sites «réactifs » favorables aux processus de corrosion. Ce dernier point est à modérer en raison de possible gênes «physico-chimique» (stérique, électrostatique,...) qui peuvent intervenir lors de l'adsorption de certains ions. La dernière origine de l'hétérogénéité de surface associée à la plasticité est la conséquence de l'émergence en surface des lignes de glissement lors de la déformation d'un cristal. Celles-ci conduisent à la formation de bandes dont la hauteur peut atteindre une centaine de nanomètres, modifiant ainsi profondément la rugosité de surface (fig. 2d). Cette rugosité de surface va affecter de façon plus ou moins importante les processus de corrosion. Ainsi à titre d'exemple, il a clairement été montré que l'énergie d'extraction des électrons dépend fortement de la rugosité de surface quelque soit l'échelle d'hétérogénéité considérée [32-34]. Il est d'autant plus facile d'extraire un électron d'un point de vue énergétique que celui-ci est moins lié au cristal et donc de favoriser certaines réactions électrochimiques propres aux processus de corrosion. Ainsi Li et al. ont clairement démontré que l'augmentation de la rugosité de surface, diminuant l'énergie d'extraction des électrons, conduisait à une augmentation des vitesses de dissolution du cuivre [34].

\section{DES ÉLÉMENTS SUR LA MÉTHODOLOGIE À ADOPTER}

Etudier l'impact des différentes échelles d'hétérogénéité décrites précédemment sur les processus de corrosion nécessite des protocoles expérimentaux particuliers. Ceux-ci ont été développés au sein de notre laboratoire sur un système modèle : nickel de haute pureté $(99,99 \%)$ - bain $\mathrm{H}_{2} \mathrm{~S}_{4}$ molaire désaéré sous argon [17, 18]. Plusieurs états métallurgiques du métal ont été mise en oeuvre afin d'aborder les effets de texture et de taille de grain dans les polycristaux, ainsi que les effets d'orientations dans les monocristaux. Les espèces majoritaires présentes dans la solution sont les ions hydronium $\mathrm{H}^{+}$ $\left(\mathrm{H}_{3} \mathrm{O}^{+}\right)$, les ions hydrogénosulfate $\mathrm{HSO}_{4}^{-}$, dans une moindre mesure les ions sulfate $\mathrm{SO}_{4}^{2-}$, et enfin les molécules d'eau. La première dissociation de $\mathrm{H}_{2} \mathrm{SO}_{4}$ étant complète pour des concentrations inférieures 
à $40 \mathrm{~mol} / \mathrm{kg}$, il n'y a pas de molécules $\mathrm{H}_{2} \mathrm{SO}_{4}$ en solution. Le $\mathrm{pH}$ de la solution est tel qu'il garantit une source abondante en hydrogène sous la forme $\mathrm{H}^{+}$. L'étude de l'influence de la plasticité sur les processus de corrosion ne peut se faire sans une préparation minutieuse de l'état de surface. Dans les travaux présentés, le polissage mécanique est suivi d'un polissage électrolytique dans un mélange $\mathrm{H}_{2} \mathrm{SO}_{4} / \mathrm{MeOH}$ [16-25]. Les conditions opératoires ont été optimisées afin de garantir un état de surface de «qualité » et reproductible (rugosité moyenne de l'ordre de grandeur du paramètre de maille du nickel). Les cellules permettant d'effectuer des mesures électrochimiques étant différentes de la cellule de polissage, le temps de transfert (moins de 2 minutes) a été optimisé afin de ne pas laisser l'oxyde natif se développer à l'air. Notons que la notion même de temps d'incubation associé à la formation de l'oxyde $\mathrm{NiO}$ est une particularité du nickel $[17,35,36]$ et qu'il existe un comportement similaire du potentiel d'abandon en fonction du temps de transfert et de la prise en masse associée à la formation d'un oxyde $\mathrm{NiO}$ lors d'une exposition sous pression d'oxygène contrôlée [17]. Ce protocole ne peut être appliqué qu'aux essais $e x$-situ, en d'autre terme pour des échantillons pré-déformés ayant subi ensuite un polissage électrolytique. Il est important de noter que cette préparation de surface ne destabilise pas les structures de dislocations développées dans les échantillons déformés. En effet il a été vérifié par des études de nano-indentation qu'à la suite d'un polissage mécanique et électrolytique, les densités et les répartitions de dislocations au voisinage de la surface $(25 \mathrm{~nm})$ sont très voisines de celles observées au MET au cœur de l'échantillon [37]. La démarche précédente permet seulement d'accéder aux effets d'une densité et d'une distribution de dislocations. La cellule utilisée pour obtenir des courbes de polarisation est une cellule conventionnelle à trois électrodes. L'électrode de référence est reliée à la solution d'analyse par l'intermédiaire d'une allonge de Luggin. Ce système permet de maintenir la température de l'électrode de référence à $20^{\circ} \mathrm{C}+/-1^{\circ} \mathrm{C}$ et de réduire la distance entre la surface métallique et l'électrode de référence de façon à minimiser la chute ohmique. Pour aborder les effets d'un état de contrainte et du changement de rugosité sur les processus de corrosion, un montage original a été développé $[21,24]$. Le but principal de celui-ci est de réaliser des mesures in-situ des paramètres mécaniques et électrochimiques avec une bonne résolution sur des machines de traction et de fatigue. Contrairement à certains travaux [38], l'éprouvette n'est pas entièrement immergée dans la solution afin d'éviter une modification du comportement mécanique par l'environnement électrochimique et donc de concentrer l'étude sur l'unique effet du champ mécanique sur les processus électrochimiques. Cette approche est similaire à celle proposée par Gutman et al. [10] bien que ces auteurs n'aient pas pu effectuer des mesures précises de la déformation en raison de la nature même de leur montage. Dans nos expériences in-situ, une seule face de l'échantillon est en contact avec l'électrolyte, cet accès est réalisé grâce à un cône en caoutchouc dont l'étanchéité est assurée par du silicone. Le montage électrochimie est traditionnel et comporte trois électrodes. La période de séchage du silicone étant longue, l'oxyde a le temps de se développer à la surface de l'échantillon. Avant l'essai électrochimique, un balayage dans le domaine cathodique est alors effectué dans le but d'éliminer cette couche d'oxyde [17, 39, 40]. L'essai électrochimique qui suit consiste en un balayage de $-150 \mathrm{mV} / \mathrm{ESH}+1000 \mathrm{mV} / \mathrm{ESH}$ à une vitesse de $0,5 \mathrm{mV} / \mathrm{s}$ [17]. L'incertitude sur la densité de courant est égale à $5 \times 10^{-5} \mathrm{~A} / \mathrm{cm}^{2}$. Au cours de ce dernier balayage, nous appliquons ou non, suivant le type d'essai, une contrainte juste avant le potentiel de $0 \mathrm{mV} / \mathrm{ESH}$. Les essais de traction sont réalisés avec une vitesse constante de $0,01 \mathrm{~mm} / \mathrm{s}$. Les mesures de déformation ont été obtenues à l'aide d'un extensomètre avec une précision de $5 \times 10^{-5}$ placés sur la face opposée hors de l'électrolyte avec des couteaux spécifiques en céramique (pour l'isolation électrique).

\section{PROCESSUS DE DISSOLUTION}

Le modèle de la surface d'un métal utilisé pour décrire le transfert d'un ion métallique du solide à la solution en une seule étape élémentaire est schématiquement composé de marches de hauteur atomique présentant des crans. 
(a)

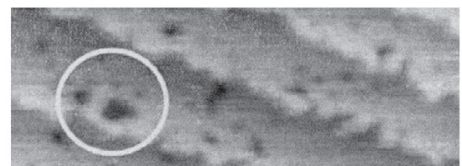

(b)

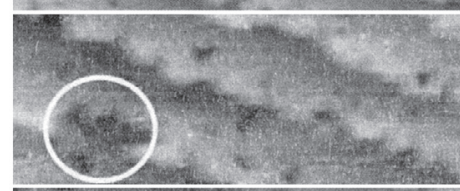

(c)

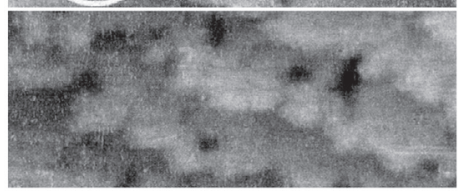

Figure 3. Dissolution localisé d'une marche mono-atomique à la surface du nickel (111) en milieu $\mathrm{H}_{2} \mathrm{SO}_{4} 0.05 \mathrm{M}$ $(-0.16$ à $-0.20 \mathrm{mV} / \mathrm{ESH})$ : (a) $36 \mathrm{~min}$, (b) $48 \mathrm{~min}$ et (c) $60 \mathrm{~min}$ [40].

L'énergie de liaison des atomes associés à ces sites est moindre que celle des atomes situés au cœur du métal. En conséquence les sites liés aux crans sont favorables énergétiquement à la dissolution anodique du métal. Ce fait a de nombreuses fois été confirmé expérimentalement par des observations directes des étapes élémentaires [40-43] (fig. 3). D'autres sites de niveaux d'énergie plus élevés, tels que les atomes situés sur les terrasses, sont aussi actifs lors de la dissolution. Des approches statistiques de ces multiples évènements ont été proposées dans la littérature [44-46]. Le modèle présenté ici restera simple au sens où il moyennera le comportement des divers sites précités.

\subsection{Mécanismes réactionnels associés à la dissolution}

L'écriture d'un mécanisme réactionnel repose souvent sur des observations concernant l'influence de la composition du bain et de la métallurgie du métal sur chaque cinétique. Dans le cas du nickel en milieu acide sulfurique les points essentiels à retenir afin de formuler le modèle sont résumés à partir des travaux les plus récents [47-53]. Dans le domaine de dissolution active, deux régimes (D-I et D-II) sont à considérer. Ces régimes ne dépendent pas de la taille de grain (plus particulièrement de la densité des joints de grains de faibles désorientations) dans le cas des polycristaux non-texturés. Le premier régime D-I est actif essentiellement aux faibles polarisations anodiques et donne lieu à de faibles densités de courant. Les cinétiques électrochimiques propres à ce régime ne dépendent pas des ions $\mathrm{OH}^{-}$mais par contre sont très sensibles à la présence des ions $\mathrm{HSO}_{4}^{-}$. Le deuxième régime D-II est clairement observé pour une gamme de potentiels plus élevés que le premier. Contrairement à ce dernier, les cinétiques électrochimiques du régime D-II ne dépendent pas de la concentration en ions $\mathrm{HSO}_{4}^{-}$mais seulement de la concentration en ions $\mathrm{OH}^{-}$. Enfin, les travaux réalisés par spectroscopie d'impédance électrochimique et par micro-balance $[54,55]$ ont clairement démontré que le mécanisme de dissolution du nickel nécessite l'échange de deux électrons comme celui du fer. Ainsi les deux régimes de dissolution seront décrits selon un mécanisme en deux étapes en accord avec des travaux antérieurs [54-56]. La dissolution du nickel se fait par un transfert ionique du métal vers la solution avec l'intervention d'un intermédiaire réactionnel de type $\mathrm{A}^{-}\left(\mathrm{HSO}_{4}^{-}\right.$dans D-I et $\mathrm{OH}^{-}$dans D-II) selon un mécanisme comprenant trois étapes $[54,57,58](\mathrm{M}=\mathrm{Ni})$ :

$$
\begin{gathered}
M_{s}^{2+}+A_{a q}^{-} \rightarrow M A_{a d}^{+} \\
M A_{a d}^{+} \rightarrow\left(M A_{a d}^{+}\right)^{*} \rightarrow M A_{a q}^{+}
\end{gathered}
$$



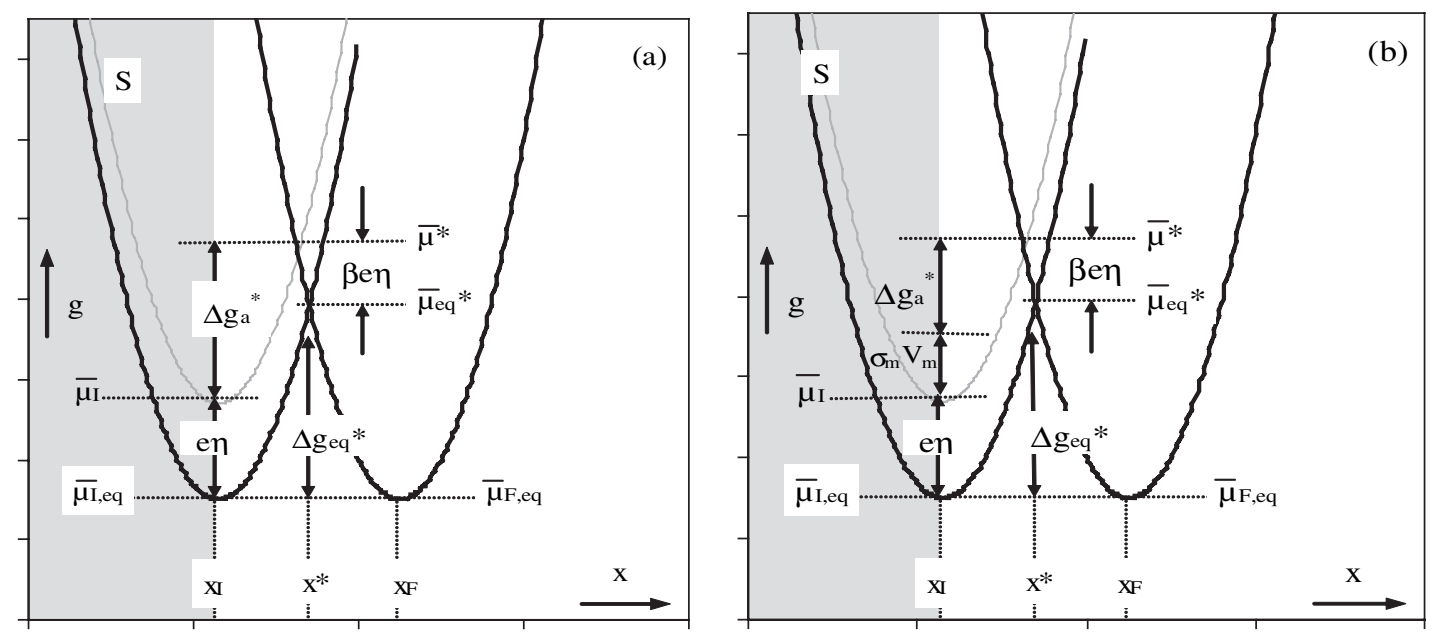

Figure 4. Évolution de l'énergie d'activation des intermédiaires réactionnels de dissolution à travers l'interface pour un état non contraint (a) et contraint (b) [22].

$$
M A_{a q}^{+} \rightarrow M_{a q}^{2+}+A_{a q}^{-}
$$

les indices $s, a d$ et $a q$ représentent, respectivement, les cations métalliques liés à la matrice solide, les espèces adsorbées et les espèces dissoutes dans la solution (hydratées).

Ainsi le processus de dissolution fait intervenir deux réactions fondamentalement différentes : une étape d'adsorption d'un intermédiaire réactionnel de type $\mathrm{A}^{-}(1)$ et une étape de transfert du complexe métallique (2) ; contribuant toutes deux à la densité de courant mesurée j. Les modèles cinétiques proposés par certains auteurs suggèrent que l'étape (2) est l'étape cinétiquement limitante [54, 55]. Nous allons donc suivre cette hypothèse dans un premier temps $(\$ 3.2, \S 3.3)$ et montrerons ses limites dans le cas des sites d'adsorption associés à la plasticité (\$3.4). Les états énergétiques successifs que traversent les cations métalliques avant de passer en solution sont représentés sur la figure 4a. L'état initial $\left(x_{I}, \tilde{\mu}_{I}\right)$ et l'état final $\left(x_{F}, \tilde{\mu}_{F}\right)$ de l'étape (2) sont rapportés sur ce diagramme. L'intersection entre les deux diagrammes d'énergie potentielle des espèces $N i A_{a q}^{+}$et $N i A_{a d}^{+}$correspond à la barrière énergétique qu'il faut franchir pour que le transfert ionique soit possible, on parle alors de complexe activé $\left(x^{*}, \tilde{\mu}^{*}\right)$. L'énergie d'activation est donnée par $\Delta g_{a}^{*}=\tilde{\mu}^{*}-\tilde{\mu}_{I}$, avec $\tilde{\mu}^{*}$ le potentiel électrochimique du complexe activé $\left(N i A_{a d}^{+}\right)^{*}$ et $\tilde{\mu}_{I}$ le potentiel électrochimique de l'ion métallique $N i A_{a d}^{+}$. Dans le cas d'une électrode polarisée, le potentiel électrochimique de l'état initial passe de $\tilde{\mu}_{I_{e q}}$ à $\tilde{\mu}_{I}$ lorsque le potentiel varie entre $E_{e q}$ et $E$ avec $\tilde{\mu}_{I_{e q}}$ et $E_{e q}$ le potentiel électrochimique et le potentiel dans les conditions d'équilibre.

L'énergie d'activation de la réaction élémentaire (2) se déduit du diagramme énergétique: $\Delta g_{a}^{*}=$ $\Delta g_{e q}^{*}-(1-\beta) F \eta$ avec $\beta$ le coefficient de symétrie et $\eta=E-E_{e q}$ la surtension de polarisation. La densité de courant $j$ est alors donnée par $j=n F v$ : où n représente le nombre d'électrons échangés, F représente la constante de Faraday et $v$ la vitesse de réaction. En appliquant les lois de la cinétique hétérogène, cette dernière dépend de la probabilité pour l'ion de franchir la barrière énergétique $\Delta g_{a}^{*}$ : $v=k_{0} \exp \left(-\Delta g_{a}^{*} / R T\right)$ avec $k_{0}$ un terme prenant en compte le nombre de sites par unité de surface sensible à la dissolution. Si nous exprimons $\Delta g_{a}^{*}$ en fonction du potentiel, la densité de courant prend la forme : $j_{2}=k_{0} n F \Gamma \theta_{i} \exp \left(-\Delta g_{a}^{*} / R T\right)=k_{2 i} n F \Gamma \theta_{i} \exp \left(-\beta_{2} E / R T\right)$ avec $\Gamma$ le nombre de sites potentiellement sensibles à l'adsorption, $\mathrm{k}_{2 i}$ et $\theta_{i}$ respectivement la constante cinétique de l'étape (2) et le taux de recouvrement de l'espèce i $\left(\mathrm{OH}^{-}\right.$et $\left.\mathrm{HSO}_{4}^{-}\right)$. Pour établir les expressions des vitesses de réactions décrites précédemment, il a été considéré que l'adsorption obéit à une isotherme de type Langmuir. Les adsorbats se comportent comme une solution idéale à la surface métallique (les 
interactions entre les entités adsorbées sont négligeables), il n'y a pas d'interaction latérale entre les espèces adsorbées et pas de modification d'énergie d'adsorption d'une espèce sur les atomes voisins [51].

\subsection{Influence d'un champ de contrainte élastique sur la dissolution anodique}

Le rôle d'un champ mécanique sur le potentiel chimique d'une espèce a été largement discuté par le passé $[11,59-67]$ dans différentes situations comme la diffusion, la métallurgie, la corrosion,... En accord avec ces travaux, le potentiel chimique des atomes du solide est supposé être modifié par la pression hydrostatique. Dans la suite, ce fait est expliqué dans le cas particulier d'un élément situé à l'interface entre un solide et un liquide selon un développement original proposé par Gutman [11]. Le travail d'origine mécanique, $\delta W$ peut être décomposé en deux parties : le travail associé au changement de volume, $\delta W_{S}$ et le travail associé au changement de forme, $\delta W_{D}: \delta W=\delta W_{\mathrm{S}}+\delta W_{D}=\sigma_{m} \delta V+$ $\bar{\sigma} \delta S . \bar{\sigma}$ représente une tension de surface, $\sigma_{m}$ la pression hydrostatique, $\delta V$ et $\delta S$ sont, respectivement, l'accroissement de volume et de surface. Le tenseur hydrostatique induit une variation de volume sans changement de forme, alors que le tenseur déviateur conduit à un changement de forme sans variation de volume. Lorsque l'on considère une réaction électrochimique à l'équilibre mettant en jeu une interface hétérogène entre un solide et un liquide, le terme d'énergie de surface est présent dans le bilan énergétique du liquide et du solide. En conséquence, ce terme n'intervient pas dans l'expression de l'enthalpie libre du système liquide/solide; seule la composante hydrostatique aura des conséquences sur l'équilibre du système. Pour déterminer la relation entre le potentiel chimique des atomes du volume d'un solide et celui des atomes de la surface participant à un processus hétérogène, il faut considérer l'état des contraintes à la surface du métal. Le potentiel chimique d'un solide peu compressible peut être exprimé en fonction du volume molaire $\left(V_{m}\right)$ et de la pression $\mathrm{P}: \Delta \mu=\int_{P_{0}}^{P} V_{m} d P=V_{m} \Delta P$ où $P_{0}$ est une pression de référence. Cette relation montre qu'une pression hydrostatique, qu'elle soit appliquée ou résiduelle, modifie le potentiel chimique du solide. Plus particulièrement, pour les atomes de la surface, lors d'une réaction de corrosion, le potentiel chimique de l'espèce i à dissoudre est alors donné par : $\mu^{i}=\mu_{0}^{i}+R T \ln \left(a_{i}\right)+\sigma_{m} V_{m}$ avec $\mathrm{a}_{\mathrm{i}}$ l'activité de l'espèce, et $\mu_{0}^{i}$ l'énergie libre standard de réaction et $\sigma_{m}=\Delta P$. $\Delta \mathrm{P}$ doit être considéré comme une valeur absolue. En effet une compression ou une traction auront le même effet sur l'énergie de Gibbs, car sa fonction associée est symétrique au voisinage de la distance d'équilibre $\left(\mathrm{r}_{0}\right)$ entre deux atomes du solide. L'influence de $\sigma_{m}$ peut être reportée sur le diagramme décrivant les états énergétiques successifs que traversent les cations métalliques avant de passer en solution (fig. 4b). Le diagramme énergétique montre que dans le cas de la présence de contraintes, une partie de l'énergie nécessaire pour la dissolution est apportée par le travail mécanique. L'énergie d'activation d'un processus de dissolution d'une électrode sollicitée mécaniquement devient alors : $\Delta g_{a}^{*}=\Delta g_{e q}^{*}-(1-\beta) F \eta-\sigma_{m} V_{m}$. Par conséquent, le rapport entre la densité de courant d'un matériau contraint et celui d'un matériau non contraint est égal à [11, 18, 21, 22, 24]: $A_{1}=\exp \left[\frac{\sigma_{m} V_{m}}{R T}\right]$. Cette approche théorique prévoit une augmentation de la densité de courant liée au processus de dissolution qui peut être évaluée pour certains métaux de structure c.f.c. (fig. 5a). A titre d'exemple, la pression hydrostatique peut augmenter la densité de courant de dissolution d'un facteur 8 pour un acier austénitique $\left(V_{m}=1,07 \times 10^{-5} \mathrm{~m}^{3} / \mathrm{mol}\right.$ et $\left.\sigma_{m}=500 \mathrm{MPa}\right)$. Il existe peu de résultats expérimentaux significatifs qui vérifient cette relation pour les métaux c.f.c., essentiellement en raison de leur trop faible limite d'élasticité. En effet, cette limite étant faible, le niveau de pression hydrostatique obtenu sous contrainte dans le régime élastique n'est pas suffisant pour valider l'expression de $\mathrm{A}_{1}$. Dans le cas du système modèle nickel- $\mathrm{H}_{2} \mathrm{SO}_{4}$, l'écrouissage a été utilisé pour augmenter la taille du domaine d'élasticité et ainsi permettre d'avoir des valeurs de $\sigma_{m}$ allant jusqu'à $66 \mathrm{MPa}$. Le rôle de $\sigma_{m}$ sur les processus de dissolution a pu être évalué grâce au rapport $\mathrm{A}_{1}$ entre la densité de courant d'un matériau contraint $\mathrm{j}\left(\varepsilon_{p}, \sigma_{m}\right)$ et celui d'un matériau non contraint $\mathrm{j}\left(\varepsilon_{p}\right)$ (essais in-situ). La seule différence entre ces deux états est le niveau de contrainte. Nous avons observé une augmentation du rapport $\mathrm{A}_{1}$ en fonction de la pression hydrostatique (fig. 5b) qui est bien décrit par l'expression théorique de $A_{1}$ avec une valeur 


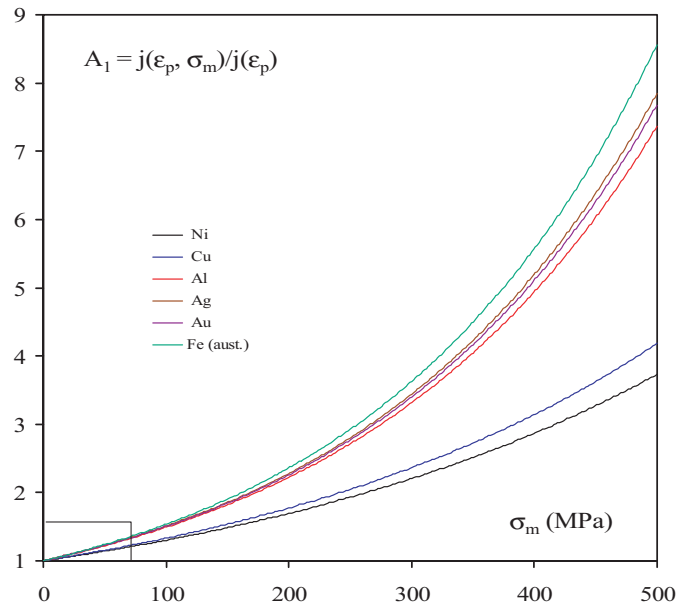

(a)

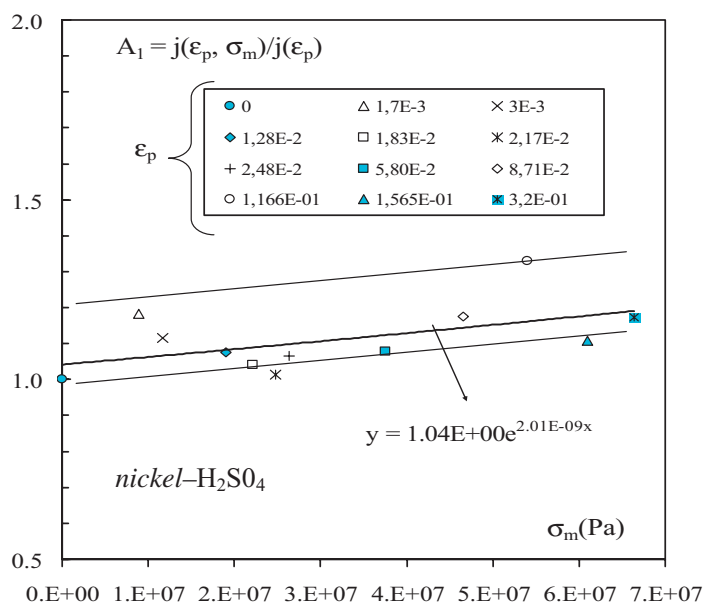

(b)

Figure 5. Évolution du paramètre $A_{1}$ pour les métaux c.f.c. (a) et du ratio $\mathrm{j}\left(\varepsilon_{p}, \sigma_{m}\right) / \mathrm{j}\left(\varepsilon_{p}\right)$ pour le nickel (b) en fonction de $\sigma_{m}[22,24]$.

de $\mathrm{V}_{m}$ égale à $4,90 \times 10^{-6} \mathrm{~m}^{3} / \mathrm{mol}$. Cette valeur est proche de celle théorique $\left(6,57 \times 10^{-6} \mathrm{~m}^{3} / \mathrm{mol}\right)$, confirmant le rôle de la pression hydrostatique sur la dissolution. Il est important de préciser que le rapport entre la densité de courant $\mathrm{j}\left(\varepsilon_{p}, \sigma_{m}\right) / \mathrm{j}\left(\varepsilon_{p}\right)$ correspond à un matériau présentant une densité de défauts cristallins différente, dépendant du niveau de déformation plastique. En conséquence, la densité de la dislocation ne modifie pas l'effet purement élastique lié à la pression hydrostatique quelles que soient la nature et la distribution des défauts cristallins. Par ailleurs, les processus d'adsorption ne sont pas affectés par la pression hydrostatique [24]. En revanche les propriétés de la couche passive semblent être modifiées car les densités de courant résiduelles sont plus faibles pour un matériau sous contrainte que pour celui non contraint dans le cas du nickel [24]. Cet effet a également été observé sur l'acier inoxydable 302 dans un bain d'acide sulfurique soumis à des contraintes de compression ou de traction [68]. Il semble d'après ces travaux qu'une contrainte appliquée de traction réduit la densité de courant et favorise la formation de la couche d'oxyde. Ce résultat peut être expliqué en termes de diffusion d'ions dans la couche d'oxyde assistée par la contrainte de traction, mécanisme favorisant la croissance de l'oxyde.

\subsection{Influence de la densité et de la distribution des dislocations sur la dissolution anodique}

La densité et la distribution de dislocations développées lors d'une traction sont susceptibles de modifier l'équilibre thermodynamique de l'interface liquide/solide. Afin d'évaluer l'intensité de cet effet, l'état de surface d'échantillons de nickel pré-déformés en traction a été préparé de façon à supprimer toute rugosité associée à l'émergence des bandes de déformation [17, 22]. Ces récents travaux démontrent clairement que le régime anodique est affecté de façon marquée par la présence de dislocations (fig. 6a) $[17,22]$. Les courants de dissolution peuvent être amplifiés d'un facteur allant jusqu'à 8 selon le niveau de déformation plastique considéré [17]. Les constantes cinétiques associées aux deux étapes du processus de dissolution ont été déterminées pour les deux intermédiaires réactionnels $\left(\mathrm{HSO}_{4}^{-}\right.$et $\left.\mathrm{OH}^{-}\right)$afin de décrire au mieux l'évolution de la densité de courant en fonction du potentiel imposé pour chacun des niveaux de déformation étudiés [18, 21]. Les coefficients $\beta$ identifiés restant constants dans toute la gamme de déformation explorée, nous avons donc évalué l'influence de la plasticité uniquement sur les constantes de vitesses $\mathrm{k}_{i}$ des réactions élémentaires 


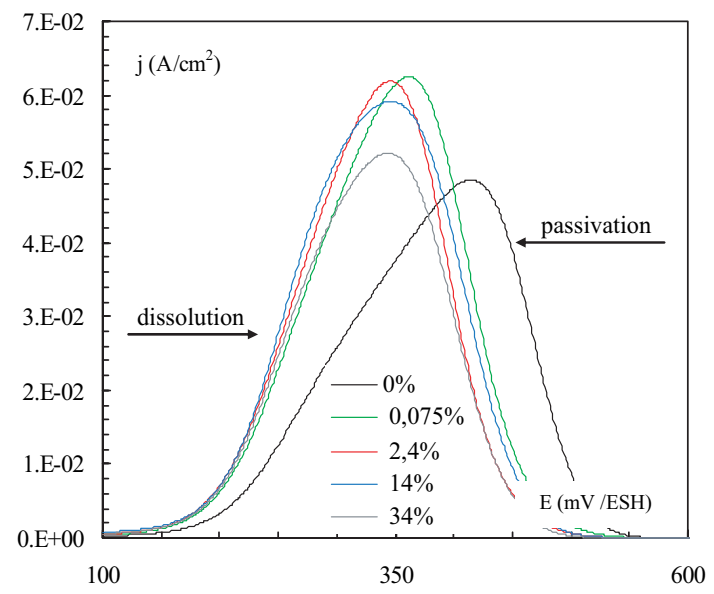

(a)

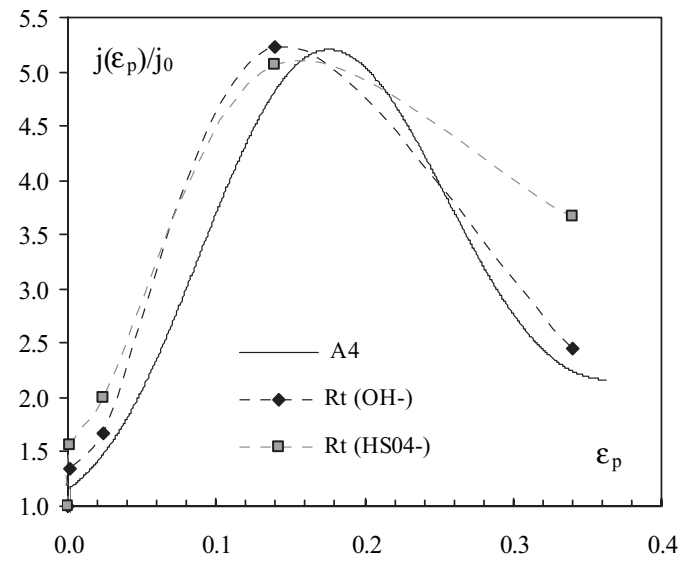

(b)

Figure 6. Évolution de la densité de courant $\mathrm{j}$ en fonction du potentiel $\mathrm{E}$ pour différent niveaux de déformation plastique (a) et dépendance du ratio $\mathrm{R}_{\mathrm{t}}$ en fonction de la déformation plastique (b) (traction de Ni-polycristallin) $[17,22]$.

(termespré-exponentiel). Cette influence est caractérisée par les rapports de ces constantes entre un état déformé et un état non-déformé $R_{i}\left(\varepsilon_{p}\right)=k_{i}\left(\varepsilon_{p}\right) / k_{i}(0 \%)$ défini pour chaque étape du mécanisme réactionnel global ( $\mathrm{i}=$ ad pour l'étape (1) et $\mathrm{i}=\mathrm{t}$ pour l'étape (2)). En ce qui concerne l'influence de la déformation sur les étapes de transfert du complexe métallique $N i A_{a d}\left(A=\mathrm{OH}^{-}\right.$ou $\left.\mathrm{HSO}_{4}^{-}\right)$vers la solution (étape (2)), nous notons une évolution en cloche des ratios $\mathrm{R}_{\mathrm{t}}\left(\mathrm{OH}^{-}\right)$et $\mathrm{R}_{\mathrm{t}}\left(\mathrm{HSO}_{4}^{-}\right)$en fonction de $\varepsilon_{p}$ (fig. 6b). En d'autres termes, la densité de courant associée à l'étape de transfert de charge augmente dans un premier temps en fonction de la déformation plastique puis diminue dans un second temps pour semble-t-il se stabiliser à des valeurs supérieures à celles d'un état non-déformé. Notons que l'effet ne semble que peu dépendre de la nature de l'intermédiaire réactionnel. Dans le cadre d'une approche thermodynamique [22] nous avons montré que l'étape de transfert du complexe métallique est fortement dépendante de l'état thermodynamique du solide considéré. L'influence de la densité et de la distribution des dislocations a été interprétée en termes de variation d'entropie du système induite par la présence de dislocations, et d'énergie élastique associée aux contraintes internes à longue distance développées par les structures de dislocations (amas, murs, cellules,... ). L'expression du terme d'amplification de la densité de courant $\mathrm{j}_{2}$ (étape (2)) obtenue est la suivante [22] :

$$
A_{4}=\left[f_{w} \exp \left(\frac{\tilde{\sigma}_{w} V_{m}}{R T}\right) \exp \left(\frac{K \sqrt{\rho_{w}}}{R T}\right)+\left(1-f_{w}\right) \exp \left(\frac{\tilde{\sigma}_{c} V_{m}}{R T}\right) \exp \left(\frac{K \sqrt{\rho_{c}}}{R T}\right)\right]
$$

avec $\mathrm{K}$ une constante, $\tilde{\sigma}_{c}$ et $\tilde{\sigma}_{w}$ les contraintes internes de nature hydrostatique dans chacune des phases de la structure hétérogène. Notons que la densité de courant à l'échelle de l'échantillon est supposée fonction, schématiquement, des densités de courant de deux phases : c et w (c.a.d. faible et forte densité de dislocations). A l'aide de l'évolution des valeurs moyennes des densités de dislocation $\left(\rho_{\mathrm{c}}, \rho_{\mathrm{w}}\right)$ et de la fraction de murs de dislocation $\mathrm{f}_{\mathrm{w}}$ déterminée en MET en fonction de la déformation plastique, il est possible d'évaluer le ratio $\mathrm{A}_{4}[22]$.

Dans le cas du nickel ces valeurs ont été obtenues sur une large plage de déformation plastique $[22,30,31]$. Les ratios $\mathrm{R}_{\mathrm{t}}\left(\mathrm{OH}^{-}\right)$et $\mathrm{R}_{\mathrm{t}}\left(\mathrm{HSO}_{4}^{-}\right)$affichent la même allure et les mêmes ordre de grandeur que le terme $\mathrm{A}_{4}$ obtenu avec le modèle thermodynamique (fig. 6b). Ainsi les processus de transfert de charge sont bien dépendants de l'état thermodynamique du système, état très sensible à la densité et la distribution des dislocations présentes dans le matériau. Il est important de souligner que la réactivité 


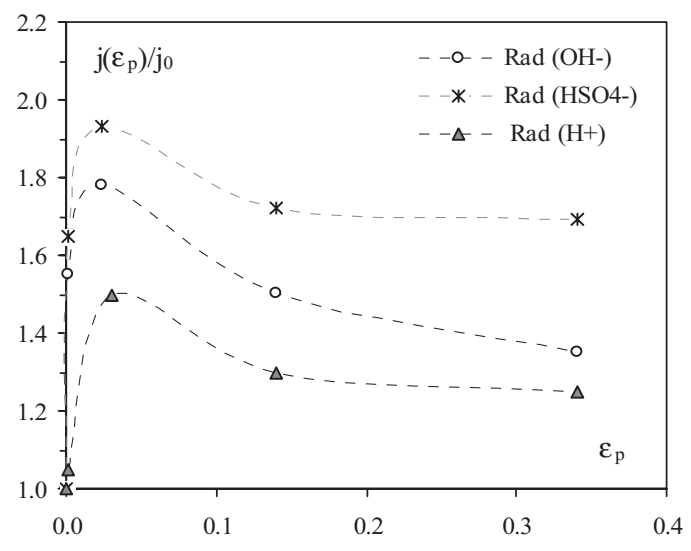

(a)

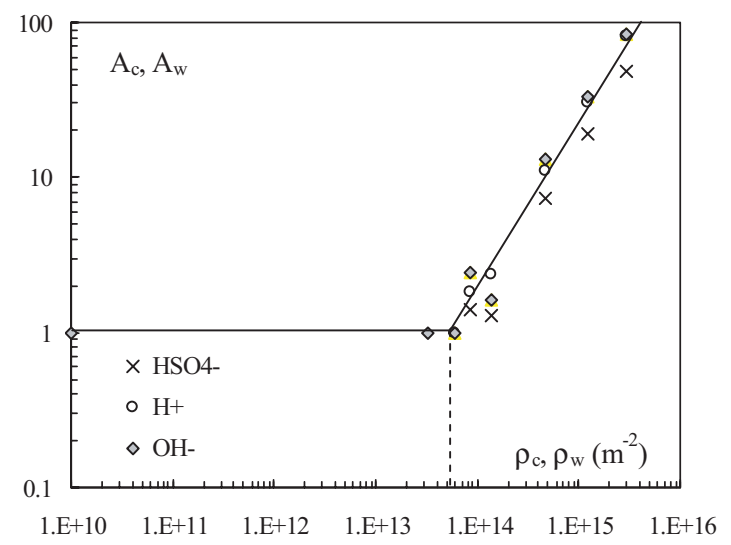

(b)

Figure 7. Évolution des ratios $\mathrm{R}_{\mathrm{ad}}$ en fonction de la déformation plastique (a). Dépendance des paramètres Ai en fonction des densités de dislocations $\rho_{\mathrm{c}}$ et $\rho_{\mathrm{w}}$ (b) (nickel polycristallin/milieu $\mathrm{H}_{2} \mathrm{SO}_{4} 1 \mathrm{M}, 300 \mathrm{~K}$ ).

de la surface augmente en fonction de la densité de dislocation et du développement d'une structure hétérogène de dislocations. Ce phénomène est partiellement restauré lors de la diminution de l'épaisseur des murs de dislocations ( $\mathrm{f}_{\mathrm{w}}$ diminue) à des niveaux de déformation élevés (stade III d'écrouissage).

\subsection{Adsorption des intermédiaires réactionnels de type $\mathrm{A}^{-}$}

Les récents travaux sur les équations cinétiques associées aux processus de dissolution ont démontré que la contribution de l'étape d'adsorption d'un intermédiaire réactionnel de type $\mathrm{A}^{-}$(étape (1)) à la densité de courant mesurée j était loin d'être négligeable [18]. Il est donc important d'évaluer le rôle de la déformation plastique sur ce processus. Ainsi, dans le cas du nickel, la densité de courant résultant de cette étape s'exprime comme : $j_{1}=k_{1 i} n F \Gamma(1-\theta) a_{A^{-}} \exp \left(-\beta_{1} E / R T\right)$ avec $\theta$ le taux de recouvrement de l'ensemble des espèces susceptibles de s'adsorber. Les travaux de Sahal et al. [22] et El Alami et al. [19] ont permis de quantifier l'effet de la déformation plastique développée en traction sur les processus d'adsorption en termes de rapport entre les constantes cinétiques $\mathrm{k}_{1 i} \mathrm{~d}$ 'un état pré-déformé et un état non-déformé, $\mathrm{R}_{\mathrm{ad}}$ (fig. 7).

Dans le cas de la dissolution, l'évolution des ratios associés aux deux ions $\mathrm{OH}^{-}$et $\mathrm{HSO}_{4}^{-}$présente la même allure. Quel que soit l'anion, l'adsorption augmente entre $0 \%$ de déformation plastique et 2,4\% pour atteindre un maximum pour ce dernier niveau. Par la suite, elle chute pour atteindre un pseudo palier tout en restant supérieure à l'état non déformé. Ce comportement conforte l'idée que l'adsorption d'anions est favorisée par les dislocations (plus de sites potentiels) mais aussi le fait que lorsque les dislocations se concentrent dans des murs il existe un mécanisme de gêne physico-chimique, qui conduit à réduire l'influence des dislocations dans le processus d'adsorption. Ce phénomène se produit quelle que soit l'espèce considérée ; en effet des résultats similaires ont été obtenus dans le cadre de l'étude des mécanismes d'adsorption de l'hydrogène (fig. 7) [19]. Dans l'hypothèse où le ratio $\mathrm{j}_{1}\left(\varepsilon_{p}\right) / \mathrm{j}_{1}(0)$ peut être assimilé à un rapport entre un nombre de sites d'un état déformé $\Gamma$ et d'un état non déformé $\Gamma_{0}$, il a récemment été proposé de décrire ce ratio en fonction de la densité de dislocations dans chaque phase de la surface « composite» :

$$
\frac{j_{1}\left(\varepsilon_{p}\right)}{j_{1}(0)}=\frac{\Gamma}{\Gamma_{0}}=1+\frac{1}{A_{0}}\left(\frac{f_{w}}{A_{w}} \sqrt{\rho_{w}}+\frac{1-f_{w}}{A_{c}} \sqrt{\rho_{c}}\right)
$$


avec $A_{0}$ un paramètre qui dépend de $\Gamma_{0}, A_{w}$ et $A_{c}$ deux paramètres dépendant de la distance entre deux sites d'adsorption situés sur une même dislocation appartenant, respectivement, à la phase dure et aux chenaux [19].

Cette équation décrit bien les évolutions observées sur la figure $7 \mathrm{a}$ pour des valeurs des $\mathrm{A}_{i}$ bien définies. Pour les niveaux de déformation qui conduisent à une distribution homogène de dislocations, le terme $A_{i}$ est égal à 1 (figure 7b), c'est-à-dire que les sites d'adsorption offerts par les dislocations sont tous disponibles. Lorsque la densité locale de dislocation atteint la valeur de $6 \cdot 10^{13} \mathrm{~m}^{-2}$, la distance entre deux sites actifs augmente linéairement avec la densité locale des dislocations, indépendamment de la phase (figure 7b). Ainsi, les fortes densités de dislocations induisent une redistribution des sites actifs en augmentant la distance entre eux, cette situation implique une diminution du nombre de sites actifs (accessibles). La figure 7b semble donc traduire le fait que les dislocations en favorisant l'adsorption peuvent entraîner un effet de gêne physico-chimique par la concentration des adsorbats. Ce dernier point est à modérer par le simple fait que nous avons supposé tous les sites de même nature, fait contestable en regard des résultats récents sur l'adsorption de l'hydrogène [23].

\subsection{Contribution de la rugosité de surface associée à la plasticité}

L'influence de la modification de la surface sur les processus de dissolution peut être mise en évidence grâce à des essais in-situ qui développent une déformation plastique à l'origine d'une rugosité liée à l'émergence des bandes de glissement $\langle\mathrm{h}\rangle$. Dans le cas du système modèle nickel/ $\mathrm{H}_{2} \mathrm{SO}_{4}$, Large et al . montrent que l'effet de ce facteur est beaucoup plus significatif que celui de la pression hydrostatique ou de la densité et de la distribution des dislocations [24]. Pour une déformation plastique de 5,8\%, la modification de la rugosité induit une modification de la valeur de la surface électro-active qui augmente la densité de courant d'environ $36 \%$ en comparaison avec un état mécanique similaire sans émergence de bandes de glissement. Récemment, Li et al. ont démontré que la morphologie de la surface peut modifier considérablement le comportement électronique caractérisé par l'énergie d'extraction des électrons et par conséquent affecter de façon marquante les processus de corrosion [34, 69]. Ces auteurs ont, en particulier, observé sur du cuivre une augmentation de $200 \%$ de la vitesse de corrosion liée à une diminution de $5 \%$ de l'énergie d'extraction des électrons résultant d'une modification de la rugosité de $16 \mathrm{~nm}$ à $275 \mathrm{~nm}$. Dans notre étude, la rugosité initiale est modifiée par l'émergence des bandes de glissement et peut atteindre une valeur proche de $26 \mathrm{~nm}$. En utilisant les travaux de Li et al. [34, 69], il est possible de déduire une augmentation de la densité de courant de $40 \%$. Cette valeur est semblable à celle obtenue expérimentalement (36\%). Par conséquent, nos résultats montrent que la rugosité liée à l'émergence des bandes de glissement favorise le processus de dissolution en relation avec l'état électronique de la surface. Cet aspect mériterait de plus amples développements.

\section{PROCESSUS D'ADSORPTION DE L'HYDROGÈNE}

L'endommagement des structures métalliques par Corrosion Sous Contrainte fait intervenir un certain nombre de processus dont la fragilisation par l'hydrogène. Il est admis que lors de ce processus, des défauts structuraux tels que les dislocations, présentes dans le matériau ou introduites par la contrainte, interagissent avec des atomes d'hydrogène, conduisant ainsi à une altération des propriétés mécaniques de la structure métallique. La première étape de ce processus, l'adsorption de l'hydrogène, fait intervenir l'interaction de l'hydrogène, contenu dans le milieu, avec la surface du métal. L'étude de l'influence des défauts de surface sur la Réaction d'Evolution de l'Hydrogène (REH) paraît donc nécessaire à une meilleure compréhension du mécanisme d'entrée de l'hydrogène dans le métal. De récents travaux sur l'effet d'une pré-déformation plastique sur l'évolution de l'hydrogène à la surface du nickel en milieu acide ont permis de montrer que la REH est convenablement décrite par le mécanisme d'adsorptiondésorption de Volmer-Heyrovsky, et d'autre part que l'état de déformation des éprouvettes modifie la densité de courant cathodique développée au cours de la REH (fig. 8) de façon significative (60\% à $303 \mathrm{~K}$ 


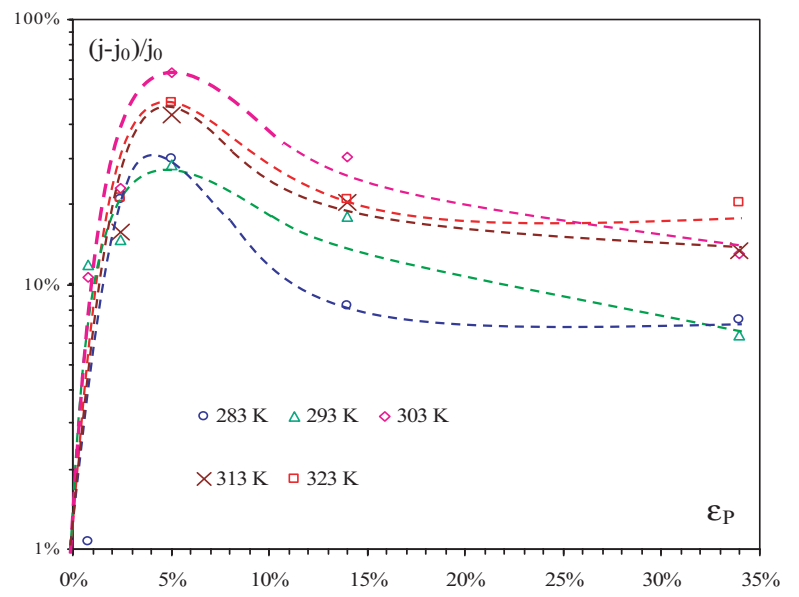

Figure 8. Différence entre les densités de courant cathodique développées sur un échantillon déformé j et un échantillon non déformé $\mathrm{j}_{0}$, en fonction du taux de déformation plastique, sur du nickel polycristallin dans $\mathrm{H}_{2} \mathrm{SO}_{4}$ $1 \mathrm{M}$, pour différentes températures, sur $[-500,-150] \mathrm{mV} / \mathrm{ESH}[20]$.

pour $\varepsilon_{p}=5 \%$ ) [20, 23]. L'étape d'adsorption (Volmer) met en jeu un transfert d'ion $\mathrm{H}^{+}$de l'électrolyte couplé à un transfert d'électron du nickel : $M+H^{+}+e^{-} \rightarrow M H_{a d s}$. La contribution à la densité de courant de cette étape peut s'exprimer de la façon suivante : $j_{V}=k_{V} F a_{H^{+}}\left(1-\theta_{H}\right) \exp \left(\beta_{V} E / R T\right)$ avec $a_{H^{+}}$l'activité des ions $\mathrm{H}^{+}, \theta_{H}$ le taux de recouvrement en hydrogène et $\beta_{V}$ le facteur de symétrie de la barrière énergétique [20,23].

Dans le cadre d'une approche thermodynamique du processus, la «constante » cinétique $\mathrm{k}_{\mathrm{V}}$ peut être formalisée en fonction du nombre de site potentiel d'adsorption $\Gamma$, de l'enthalpie $\Delta H_{V}^{*}$ et de l'entropie $\Delta S_{V}^{*}$ associée à l'énergie d'activation : $k_{V}=\left(\kappa k_{B} T / h\right) \Gamma_{V} \exp \left(\Delta S_{V}^{*} / R\right) \exp \left(-\Delta H_{V}^{*} / R T\right)$ avec $h$ la constante de Plank, et $\kappa$ un coefficient de transmission qui caractérise le franchissement de la barrière énergétique par le complexe activé [23]. Les courbes de polarisation cathodique réalisées en température permettent d'accéder à $\Gamma$ et $\Delta H_{i}^{*}$ en supposant connue et indépendante de la déformation plastique $\Delta S_{V}^{*}$ $\left(-542 \mathrm{~J} \mathrm{~mol}^{-1} \mathrm{~K}^{-1}\right)[23,25]$.

Le nombre de sites d'adsorption de l'hydrogène peut être corrélé à la densité de dislocation totale développée lors d'une traction simple sur des polycristaux (fig. 9a). Celui-ci augmente en fonction de la densité de dislocation lors des stades I et II d'écrouissage et diminue en stade III, lorsque la densité de dislocations dans les murs diminue (restauration). Dans ce dernier cas, malgré des densités de dislocations assez similaires entre les stades II et III $\left(\rho \approx 3 \cdot 10^{14} \mathrm{~m}^{-2}\right)$, le nombre de sites supplémentaires associés à la plasticité est bien inférieur en stade III qu'en stade II. Ce dernier point confirme l'idée que l'affinement des murs de dislocations pour les taux de déformation importants conduit à des gênes physico-chimiques qui réduisent le nombre de sites potentiels d'adsorption. Les résultats conduits sur mono-cristaux sont assez similaires à ceux obtenus sur des polycristaux (fig. 9a). Indépendamment du taux de déformation, $\Gamma$ obtenu pour l'orientation $\langle 153\rangle$ est supérieur à celui du polycristal non-texturé en raison d'une densité de marche initiale importante (de l'ordre de $5 \mathrm{~nm}^{-1}$, fig. 1a). L'analyse en termes d'énergie d'activation du processus d'adsorption montre que cette énergie augmente lors des premiers stades de déformation (I et II), c'est-à-dire que l'adsorption d'hydrogène devient plus difficile lors d'une augmentation de la densité de dislocation.

Il a été suggéré une possible implication des processus d'absorption pour expliquer la désactivation progressive de la surface vis-à-vis de l'adsorption [70-73]. Cette désactivation est souvent attribuée à la formation d'un hydrure. Mais nos conditions expérimentales n'étant pas suffisamment sévères pour former un hydrure structuré à la surface du nickel [19], nous pouvons cependant considérer que de 

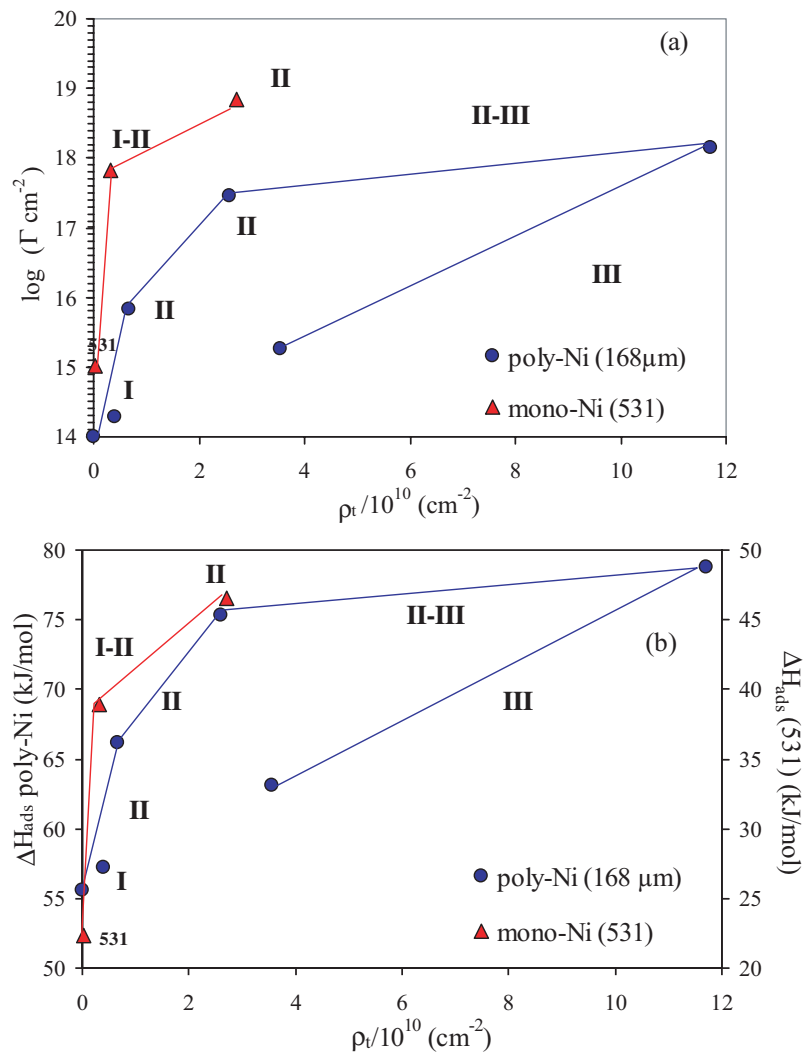

Figure 9. Évolution de $\Gamma$ de l'enthalpie $\Delta H_{V}^{*}$ en fonction de la densité totale de dislocation $\rho_{\mathrm{t}}$ (nickel polycristallin et monocristallin (153) dans $\mathrm{H}_{2} \mathrm{SO}_{4} 1 \mathrm{M}$ ) [25].

l'hydrogène pénètre dans les toutes premières couches de la surface [74]. Dans ce cas, une organisation, voire une phase se formant en sub-surface modifierait la structure électronique du nickel [75] et rendrait toute nouvelle interaction nickel/hydrogène moins favorable [76, 77]. Si l'on considère l'idée communément admise que les concentrations de dislocations constituent des circuits préférentiels pour la diffusion et la ségrégation d'hydrogène, l'augmentation de ce type de circuits induirait une augmentation de l'hydrogène absorbé dans le matériau. Sur nos échantillons de nickel déformés durant les stades I et II, la densité de dislocations s'accumulant dans les murs des cellules rend ces murs de plus en plus épais. Ceci pourrait être à l'origine d'une absorption d'hydrogène de plus en plus importante, et qui pourrait par conséquent rendre progressivement toute nouvelle interaction nickel/hydrogène moins favorable. Cette explication permettrait de justifier que l'énergie d'activation du processus d'adsorption de l'hydrogène augmente avec la déformation plastique pendant les stades I et II, et diminue pendant le stade III (où les murs des cellules s'affinent et la densité totale de dislocations diminue). Cependant, cet effet désactivant est masqué par l'augmentation considérable du nombre de sites actifs, de sorte que l'augmentation de la déformation plastique durant les stades I et II a globalement un effet activant sur le rendement de la REH. Le rôle de la rugosité de surface (émergence de bandes de déformation) sur l'adsorption de l'hydrogène est un domaine peu étudié. Les premiers résultats sur notre système modèle semblent démontrer que les densités de courants cathodiques sont amplifiées par la présence d'une rugosité de l'ordre de 50 à 100 nm développée lors d'essais de traction sur monocristaux orientés en glissement multiple (001) [77]. 


\section{BILAN ET PERSPECTIVES}

Les travaux présentés dans cette synthèse contribuent à la compréhension des mécanismes d'interaction entre le processus de corrosion et la plasticité des matériaux métalliques. Les diverses techniques expérimentales explorées démontrent l'importance de prendre en compte les différentes expressions de la déformation plastique (densité et distribution des dislocations, l'émergence des bandes de glissement, champ de contrainte) afin d'élucider l'effet de l'état mécanique sur les processus de corrosion. La déformation plastique a pour effet de favoriser les processus de dissolution et d'adsorption d'espèces qui parfois conduisent à la passivation du matériau. Ces deux mécanismes antagonistes peuvent conduire à un effet bénéfique ou néfaste vis-à-vis de la corrosion de la déformation plastique selon le poids d'un processus par rapport à l'autre. La prise en compte de l'aspect énergétique et statistique du couplage dislocation/dissolution a permis de développer un modèle cinétique rendant compte du comportement anodique du nickel polycristallin quel que soit son niveau de déformation. En revanche l'adsorption d'intermédiaire réactionnel et son interaction avec la plasticité n'est pas à négliger dans la description du processus de dissolution. De plus l'augmentation de la rugosité de la surface électro-active liée à l'émergence des bandes de glissement favorise le processus de dissolution en relation avec l'état électronique de la surface. La plasticité n'affecte pas seulement le nombre de sites d'adsorption lors de la REH mais aussi les états énergétique. La transition entre l'adsorption et l'absorption d'espèces réactives est essentielle si l'on veut comprendre l'amorçage d'un endommagement au voisinage d'une surface sollicitée mécaniquement. En effet, cette transition peut conduire à des modifications importantes de l'état énergétique du solide au voisinage de la surface. Dans cette optique, des techniques électrochimiques de type pulsées et de perméations sont en cours de développement. Les premiers résultats obtenus sur monocristaux, confrontés à ceux des polycristaux, démontrent clairement l'importance d'avoir une bonne description des transitions d'échelles. Les modèles d'homogénéisation, couramment développés dans le cadre de la modélisation du comportement mécanique d'agrégats polycristallin, semblent être de bons candidats pour réaliser cette étape.

\section{Remerciements}

Les auteurs tiennent à exprimer leurs sincères remerciements à B. Peraudeau et S. Cohendoz pour leurs soutiens techniques associés aux nombreux montages développés au sein du LEMMA. L'ensemble des observations en microscopie électronique ont été réalisées au sein du Centre Commun d'Analyse de l’Université de La Rochelle.

\section{Références}

[1] Birnbaum H. K., Sofronis P., Mater. Sci. Eng. A 176 (1994) 191.

[2] Château J.P., Delafosse D., Magnin T., Acta Mater. 50 (2002) 1507.

[3] Ford F.P., Taylor D.F., Andresen P.L., Ballinger R.G. EPRI NP-5064M, 1987.

[4] Ford FP. In Corrosion sous contrainte. Phénoménologie et mécanismes. EDP Science (1990) 307.

[5] Ford F.P., Corros. Sci. 52 (1996) 375.

[6] Saito K., J. Kuniya, Corros. Sci. 43 (2001) 1751.

[7] Galvele JR., Corros. Sci. 27 (1987) 1.

[8] Galvele J.R., Corros. Sci. 36 (1994) 901.

[9] Garz I., Häfke U., Corros. Sci. 11 (1971) 329.

[10] Gutman E.M., Solovio G., Eliezer D., Corros. Sci. 38 (1996) 1141.

[11] Gutman E.M., in Mechanochemistry, Singapore: World Scientific (1994) 68.

[12] Despic A.R., Raicheff R.G., Bockris J.O’M. J. Chem. Phys. 49 (1968) 926.

[13] Devanathan M.A.V., Fernando M. J. Electrochim. Acta 15 (1970) 1623.

[14] Garz I. and Häfke U. Corros. Sci. 11 (1971) 329. 
[15] Bachir A.B. Mém. Scie. Revue Métall., 1975; Janvier: 61

[16] Sahal M., Creus J., Huvier C., Sabot R., Feaugas X. in Proc. of the $2^{\text {nd }}$ Inter. Conf. on EDEM 2003.

[17] Sahal M., Creus J., Sabot R., Feaugas X., Scripta Mat. 51 (2004) 869.

[18] Sahal M., Thèse de Doctorat, Université de La Rochelle, 2006.

[19] El Alami H., Creus J., Feaugas X., Electrochim. Acta 51 (2006) 4716.

[20] El Alami H., Creus J., Feaugas X., Matériaux 2006, Dijon, France, 2006.

[21] Large D., et al., Matériaux 2006, Dijon, France 2006.

[22] Sahal M., Creus J., Sabot R., FeaugasX., Acta Mat. 54 (2006) 2157.

[23] El Alami H., Creus J., Feaugas X., Electrochim. Acta 52 (2007) 4004.

[24] Large D., Sabot R., Feaugas X., Electrochim. Acta, 2007, to be published.

[25] El Alami H., Creus J., sabot R., Feaugas X., EDEM07, 2007.

[26] Oura K., et al. Surface Science, an introduction, Springer, 2003.

[27] Feaugas X., Haddou H., Phil. Mag. 87 (2007) 989.

[28] Huvier C., Feaugas X., communication privée 2004.

[29] Budevski E., Staikov G., Lorenz W.J., Electrochemical phase formation and growth, VCH, 1996.

[30] Feaugas. X., Acta Mat. 47 (1999) 3617.

[31] Feaugas X., Haddou. H., Metall. Trans. A 34A (2003) 2329.

[32] Li W., Li D.Y., Appl. Surf. Sci. 240 (2005) 388.

[33] Li W., Cai M., Wang Y., Yu S., Scripta Mat. 54 (2006) 921.

[34] Li W., Li D.Y., Acta Mater. 54 (2006) 445.

[35] Mitchell D.F., Graham M.J. Int Corrosion conf. 6 (1981) 18.

[36] Pope T.D., Bushby S.J., Griffiths K., Norton PR. Surf Sci. 258 (1991) 101.

[37] Delobelle P., Haddou H., Feaugas X., Matériaux 2002 Tours : 21-25 avril, 2002.

[38] Laird C., Farrington G.C., Li Y.F., Acta. Metall. Mater. 41 (1993) 693.

[39] Zuili D., Maurice V., Marcus P., J. Electrochem. Soc., 14 (2000) 1393.

[40] Scherer J., Okbo B.M., Magnussen O.M., Electrochim. Acta 48 (2003) 1169.

[41] Vogt M.R., Lachenwitzer A., Magnussen O.M., Behm R.J, Surf. Sci. 399 (1998) 49.

[42] Müller P. Satoru A., Yamada T., Itaya K., J. of Electroanl. Chem. 467 (1999) 282.

[43] Broekmann P., Hai N.T.M., Wandelt K., Surf. Sci. 600 (2006) 3971.

[44] Allgaier W. Heusler K.E., J. Appl. Electr. 9 (1979) 155.

[45] Folleher B., Heusler K.E., J. Electroanal. Chem. 180 (1984) 77.

[46] Keddam M. in Corrosion mechanisms in theory and practice, ed. P. Marcus, Marcel Dekker 2002:97.

[47] Gregori J., Garcia-Jareño J.J., J. Solid State Electrochem., 9 (2005) 83.

[48] Itagaki M., Nakazawa H., Watanabe K., Noda K., Corros. Sci., 39 (1997) 901.

[49] Zuili D., Maurice V., Marcus P., J. Electrochem. Soc., 14 (2000) 1393.

[50] Barbosa M.R., Bastos J.A., Garcia-Jareno J.J., Vicente F., Electrochim. Acta 44 (1998) 957.

[51] Protopopoff E. and Marcus Ph., Electrochim. Acta 51 (2005) 408.

[52] Kitakatsu N., Thèse de Doctorat, Université Paris 6, 1997.

[53] Seyeux A., Maurice V., Klein L. H., Marcus P., Proc. of $9^{\text {th }}$ Symp. of Passivity, Paris 2005.

[54] Gregori J., Garcia-Jareño J.J., J. Sol. State Electrochem., 9 (2005) 83.

[55] Itagaki M., Nakazawa H., Watanabe K., Noda K., Corros. Sci., 39 (1997) 901.

[56] Jouanneau A., Keddam M., Petit M. C., Electrochim. Acta 21 (1976) 287.

[57] Barcia O.E., Mattos O.R., Electrochim. Acta 35 (1990) 1601.

[58] Sato N., Electrochemistry at Metals and Semiconductor Electrodes, Elsevier Science B.V., 1998.

[59] West.M., Metal Science, nov. (1980) 534.

[60] Johnson W.C., Cahn J.W., Scripta Metall. 15 (1981) 1259.

[61] Larché F.C., Cahn J.W., Acta Metall. 33 (1985) 331.

[62] Qiao L., Mao X., Acta Metall., 43 (1995) 4001. 
[63] Sofronis P., J. Mech. Phys. Sol. 43 (1995) 1385.

[64] Stashchuk M.H., Mater. Sci., 36 (2000) 54.

[65] Liang J., Suo Z., Inter. Sci., 9 (2001) 93.

[66] Monroe C., Newman J., J. Electrochem. Soc. 151 (2004) A880.

[67] Li W., Li D.Y., Appl. Surf. Sci. 240 (2005) 388.

[68] Navaï F., J. Mater. Sci. 30 (1995) 1166.

[69] Li W., Li DY., J. Chem. Phys. 122 (2005) 064708.

[70] Rommal H.E.G., Moran P.J., J. Electrochem. Soc. 132 (1985) 325.

[71] Rommal H.E.G., Moran P.J., J. Electrochem. Soc. 135 (1988) 343.

[72] Huot J.-Y., Brossard L. J. Appl. Electrochem. 20 (1990) 281.

[73] Huot J.-Y., Brossard L. J. Appl. Electrochem. 21 (1991) 508.

[74] Highfield J. G., Claude E., Oguro K. Electrochim. Acta 44 (1999) 2805.

[75] Martinez S., Meticos-Hukovic M., Valek L. J. Mol. Catal. A 245 (2006) 114.

[76] Smithson H., et al., Phys. Rev. B 66 (2002) 144107.

[77] Nilsson A., et al., Catal. Lett. 100 (2005) 111.

[78] El Alami H., Creus J., Feaugas X., Huvier C., Conforto E., communication privé, 2007. 